\title{
ESTABLECIMIENTO DE NORMAS PARA UN BUEN USO DE LA COMUNICACIÓN INTERNA Y SU IMPACTO EN LA SATISFACCIÓN DEL CLIENTE
}

DEVELOPMENT OF RULES FOR A GOOD USE OF INTERNAL COMMUNICATION AND ITS IMPACT ON CUSTOMER SATISFACTION

MAG. CRA. MA. MACARENA SUÁREZ BLANCO'

Recibido: 22 de septiembre de 2019 Aceptado: 1 de noviembre de 2019 



\title{
ESTABLECIMIENTO DE NORMAS PARA UN BUEN USO DE LA \\ COMUNICACIÓN INTERNA Y SU IMPACTO EN LA SATISFACCIÓN DEL CLIENTE
}

\author{
DEVELOPMENT OF RULES FOR A GOOD USE OF \\ INTERNAL COMMUNICATION AND ITS IMPACT \\ ON CUSTOMER SATISFACTION
}

Mag. Cra. Ma. Macarena Suárez Blanco

Palabras clave: Mapa interno, clientes, Derechos Humanos, comunicación organizacional, objetivos

Keywords: Internal map, customers, Human Rights, organizational communication, objectives

\section{RESUMEN}

En este artículo se propone demostrar la relevancia que tiene la aplicación de una comunicación eficaz dentro de la empresa, para los colaboradores y clientes, con la cual se pueda llegar a su mapa interno. Con este fin, nos proponemos estudiar bibliografía de los últimos años especializada en la temática, para comprender las necesidades y reacciones de los colaboradores frente a la comunicación organizacional y también la captación de los clientes por medio de ello. Se desea saber si realmente se puede captar clientes por medio de un ma- 
yor cuidado de los derechos humanos de los trabajadores. En conclusión, se visualiza la relevancia de poder establecer mecanismos de motivación y comunica- ción eficaz con los trabajadores, para que pueda repercutir en el cumplimiento de las aspiraciones de los clientes.

\section{ABSTRACT}

This article aims to demonstrate the relevance of the application of effective communication within the company, for employees and customers, with which it could be possible toreach its internal map. To this end, we intend to study specialized bibliography from recent years on the subject, to understand the needs and reactions of employees in face of organizational communication and also the acquisition of customers through it. This article wants to unveil if it is really achievable to attract customers through greater care of workers. In conclusion, the relevance of generating key alliances within the company with the workers is visualized, so it can have an impact on the clients' aspirations.

\section{INTRODUCCIÓN}

El mundo en que vivimos es tan complejo; que necesitamos simplificarlo, para entenderlo o filtrar la información. Los seres humanos recibimos la información por medio de los cinco sentidos, a saber: vista, oído, gusto, tacto y olfato. Los mismos se agrupan, de acuerdo a la programación neuro-lingüística, en tres: auditivo, visual y cinestésico.

Como puerta de la percepción, los clientes utilizamos los sentidos detallados anteriormente, siendo los únicos puntos de contacto con el exterior, y los mecanismos neurológicos por medio de los cuales interpretamos el mundo. (Huxley, 2009).

Hay una infinidad de posibles impresiones sensibles, pero, solo percibimos una parte pequeña de estos; lo que obtenemos, es filtrado por nuestro mapa interno que está compuesto por percepciones, motivaciones, cultura, valores, intereses, creencias, lenguajes, etcétera. Por eso, cada uno obtenemos nuestra realidad única, cuando actuamos por lo que percibimos, nuestro modelo del mundo. (O'Connor, J., Seymour, J., 2007). 
Robbins (2013), expresa que "Una cultura firme proporciona estabilidad a una organización... Y para algunas organizaciones, incluso llega a convertirse en un obstáculo importante para el cambio" (pp. 312).

Para poder entender el exterior y simplificarlo tomamos diferentes dimensiones que podemos generalizar en:

- Distorsión son aquellos procedimientos por los cuales vamos a transformar y alterar lo que vemos y sentimos por nuestra experiencia.

- Omisión nosotros prestamos atención de forma selectiva, es por eso que vamos a atender cosas que nos Ilamen la atención e ignorar aquellas que no sean de nuestro agrado.

- Generalización es cuando tomamos esas percepciones y las categorizamos de acuerdo a nuestros modelos mentales. (Carrión, S., 2014, p. 23).

A pesar que conscientemente somos capaces de retener una cantidad limitada de información, inconscientemente podemos adquirir una mayor cantidad de información.

Lo consciente viene dado, por ejemplo, al estar leyendo este artículo, mientras que lo inconsciente sería el aroma que hay en la habitación donde estamos o el ruido de los pájaros que sentimos mientras leemos.

Creemos que la mayor parte de nuestras acciones las tomamos de forma consciente, no obstante, las decisiones inconscientes son las que regulan nuestros comportamientos.

Aunque no lo notemos el cerebro continuamente está tomando decisiones, por eso, es importante entrenarlo con sentimientos positivos, estos y las emociones afectan al cerebro. Por día, el cerebro tiene más de 65.000 pensamientos, que generalmente son los mismos. (Rodriguez, 2016, pp. 56-57).

Los procesos de comunicación son inherentes a la operativa de la empresa, configurándose tal cual es, conformando su identidad, y de la mano con ello, proyectando su imagen.

Comunicar supone transparentar y ser transparente, permite a la organización ganar en flexibilidad, lo que genera un pilar básico para poder transformarse teniendo en cuenta las condiciones sociales, ambientales y políticas; además de bregar por sus intereses propios, mejorando así la sostenibilidad en el tiempo de la empresa.

Este proceso de comunicación se visualiza en cuatro aspectos clave:

- En los procesos administrativos: como factor primordial de la coordinación y mejora continua por medio del aprendizaje compartido.

- En las relaciones de trabajo y en la interacción de los individuos, que son parte de la empresa. 
- $\quad$ Provocando diversos tipos de vínculos con consumidores, ya que todos los procesos de servicios son esenciales.

- Por otro lado, elaborar informes va a cooperar a la proyección de la orga- nización como cuerpo (corporación)

y por medio de esto, de una forma u otra, se establecen lazos de interacción directa o indirecta con los stake-holders.

\section{DESARROLLO}

La identidad es la respuesta a la pregunta ¿quiénes somos?, de acuerdo a Barnett y otros(2006), se define como el corazón de la firma, es un elemento intangible, clave para cualquier organización, que la auto-define, desarrolla sus temas estratégicos, recursos y las capacidades que requiere para su mantenimiento y desarrollo. A la vez, es de gran utilidad para visualizar en qué forma la empresa va a establecer y hacer perdurables en el tiempo sus ventajas competitivas. (Fiol, 1991).

La información, para que sea útil y pueda generar ventajas a la empresa, debe ser completa, oportuna y fiable. Es decir, que de acuerdo a Burch y Strader (citado en Pérez y Coutín, 2005), debe ser accesible, comprendida, precisa, relevante, clara, puntual, flexible, verificable y cuantificable. (Recurso electrónico).

Por la facilidad de asociación y entendimiento del tema abordado en este trabajo, se utilizan y contextualizan ejemplos específicos del ámbito hotelero.
Por ejemplo, probablemente dentro de la identidad de una cadena hotelera se buscará brindar experiencias inigualables a los huéspedes, una atención integral sustentada por los valores de la organización, por un servicio diferente y variado. La experiencia en un servicio es fundamental, la misma tiene como pilares la atención al cliente, la amabilidad y empatía hacia el mismo.

En hotelería cobra especial relevancia dado que; en muchas ocasiones es la primera vez que el huésped recorre un lugar y por ende, conoce un destino.

Por eso es tan importante tener un mayor conocimiento del lugar para explicar al huésped lo mejor posible, ya que, generalmente está "perdido" y tiene tiempo limitado para recorrer un sinfín de lugares. De hecho existen muchos otros locales que desconoce dado que, si bien, generalmente se le informa sobre el destino al que va a arribar, muchas veces piensa que no es accesible o desconoce sus aspectos más relevantes o bien 
siente que su presupuesto económico y temporal no es suficiente.

Si se analiza el total de los clientes que tienen una queja importante en un establecimiento hotelero de acuerdo a un estudio del Instituto de Investigación de Programas Técnicos de EEUU, el $91 \%$ no va a volver a hospedarse en el mismo lugar, mientras que si se le soluciona el problema rápidamente, el $82 \%$ considera que volverá.

Esta reducción en las quejas de los clientes y subsanación de los problemas va a pasar de una baja de clientes de un $91 \%$ a un $18 \%$. Mientras que si se siguen reduciendo las quejas este índice de baja de clientes va a disminuir a menos de un $5 \%$. (Kotler, et al., 2011).

Y no siempre es así. Por eso, es tan importante la primera impresión, conocida como el momento de la verdad. Si ese momento, no es bueno, va a causar una mala impresión, lo que conlleva una evaluación negativa del mismo o no tan positiva como hubiera esperado.

La estructura superficial es el conjunto de símbolos o signos para la escritura, y las palabras para la exposición que hacemos de forma oral. Mientras que la estructura profunda es el significado que presenta cada uno de los símbolos y sonidos que efectuamos, es decir, la experiencia interna (Carrión, 2011). La primera es aquello que se ve o se siente por un tercero, mientras que la segunda no siempre es expresada.

Estos aspectos no siempre son considerados por los gerentes o encargados de atención al público en muchos hoteles de Uruguay, por ejemplo, lo que ocasiona divergencias entre lo que los clientes dicen y sienten, generando experiencias negativas 0 no totalmente positivas.

Una mayor aproximación nos permitirá comprender, predecir y mejorar el comportamiento de los sujetos de una organización, en temas tales como motivación, liderazgo, poder, comunicación, procesos en los cambios, conflicto, entre otros. (Robbins y Judge, 2013).

De acuerdo al análisis de Joan Costa (imagen e imágenes en acción p. 52-70), la imagen corporativa no está dentro del entorno físico, sino que se conforma de la memoria latente de los individuos que idealizan e interpretan la organización. Está compuesta por una serie de valores, visiones y atributos que conforman la empresa y la asocian a determinado estereotipo, en el que las imágenes visuales tienen un papel muy importante. Se entiende como un instrumento estratégico, no es una moda.

Intenta responder a las preguntas: ¿cómo ven a la empresa externamente?, ¿es lo que se quiere proyectar? Se conforma por varias causales tales 
como la percepción del servicio, que son propias del individuo (para él puede ser correcto pagar 100 dólares por el servicio de dos habitaciones, pero le parecería demasiado pagar 200 dólares por ese servicio).

La inducción y deducción de cómo va a ser el servicio, se basa en su experiencia (distorsión) y en la generalización del mismo. Las experiencias que se dilucidan durante el uso del mismo, asociado también con las vivencias, el conocimiento del servicio que espera y el precio que desea pagar por ese servicio.
La imagen externa es clave en el proceso de cambio de identidad, ya que es el feedback que los miembros de la organización reciben de los stake-holders externos.

Se presenta una relación indisoluble entre identidad e imagen dentro de la perspectiva interna sobre lo que piensan los integrantes, formas de identificarse, cómo creen que se percibe la empresa y la forma en qué les gustaría que fuera vista; por eso, es fundamental pensar las estrategias de forma amplia considerando la gerencia social y los derechos humanos.

\section{GERENCIA SOCIAL Y DERECHOS HUMANOS}

El discurso de los Derechos Humanos no es nuevo, sino que viene a lo largo de la historia, tomando en cuenta diferentes normas de carácter internacional en la materia, adoptando diferentes legislaciones, dependiendo de las naciones. En este sentido, se llega a las preguntas: ¿dónde aparece la empresa y por qué?, ¿qué función tiene?

Una definición de justicia del derecho es darle a cada uno lo suyo, lo que supone es comprometerse a que se generen relaciones de crecimiento, con cada miembro de la empresa, con sus diferencias y con exigencias acordes a los mismos. (Rodriguez, 2016, pp. 126-127).
La gerencia social pasa de "un proyecto para el otro", a "un proyecto con el otro". Lo que supone realizar un estudio profundo de las necesidades y expectativas del otro, poniendo en el eje a los stake-holders. Supone gerenciar una amplia gama de enfoques de la economía social, enfocándose en lograr transformaciones sociales. (Volpentesta, 2011, 105-108).

El Pacto Global de la ONU surge el 31 de enero de 1999, uniéndose Uruguay el 5 de marzo de 2010; es una asociación de carácter voluntario, por medio de la cual las organizaciones y empresas se comprometen a alinear sus 
estrategias empresariales a 10 principios universales, los cuales se enfocan en cuatro áreas temáticas, a saber: derechos humanos, medioambiente, anti-corrupción y estándares laborales. (Deres Uy, s/f, recurso digital).

De acuerdo al Pacto Global "Las empresas deben apoyar y respetar la protección de los Derechos Humanos fundamentales, reconocidos internacionalmente, dentro de su ámbito de influencia". (Pacto Global, 2017, op. cit, recurso electrónico).

Lo que supone que las organizaciones deberán actuar con due diligence, para no dañar los Derechos Humanos, y poder abordar de forma eficaz, disminuyendo, mitigando o previniendo los impactos negativos que puede causar a ello. Esto supone, crear acciones por propia voluntad organizacional, que contribuyan positivamente al cumplimiento de los Derechos Humanos, invirtiendo, participando o promoviendo acciones colectivas y alianzas estratégicas.

El mismo Pacto Global expresa que debe haber una relación entre el imperativo moral para actuar de forma responsable y los derechos humanos.

Si bien, en muchas ocasiones las empresas adoptan códigos voluntarios, no siempre son adecuados, ni eficaces para evitar el abuso en Derechos Humanos; ya que, muchas veces, estos análisis internos, se ven sesgados por no evaluar objetivamente a la empresa. (Giner, 2008, pp. 68-70).

Desde la visión de la empresa, se puede decir que no hay estándares claros, en base a los cuales, las organizaciones puedan ajustar sus actividades, como tampoco, se encuentran recursos directos donde puedan acudir las víctimas. (Tangarife-Pedraza, 2008, pp. 145-146).

Los elementos fundamentales que la empresa debería adoptar, como proceso, en este sentido son:

- Evaluar los impactos causados en los Derechos Humanos, teniendo en cuenta sus actividades y relaciones.

- Generar políticas de proyección de los Derechos Humanos internas a la empresa, estando integrados a todas las funciones y políticas empresariales.

- Seguimiento del proceso: lo que supone generar incentivos, recomendaciones, des incentivos para poder garantizar el cumplimiento de estas prioridades.

Comunicar los procesos, esta información generada debe fluir por la organización interna y externamente.

- Compensar daños causados, en caso de generar acciones adversas a los Derechos Humanos la empresa ha de subsanar estos daños. (Pacto Mundial, red española, recurso electrónico). 
La buena reputación de las empresas, se gana adoptando buenas prácticas y comunicándolas interna y externamente, para conseguir aprobación y retroalimentación. Es importante, involucrar a los miembros de la empresa y a los afectados para el logro de los objetivos, por tanto, es fundamental que la comunicación interna fluya de forma efectiva por todas las áreas de la organización. (Cortina, 2005 p. 2).

\section{ESTRATEGIA DE COMUNICACIÓN INTERNA PARA MEJORAR LA MOTIVACIÓN INTERNA}

Muchas veces, en las grandes empresas e incluso en las pequeñas, es muy difícil que todos los miembros de la organización se conozcan personalmente, por tanto, se ven limitadas las redes de contacto a un reducido número de compañeros.

Cada uno como individuos miembros de la organización, tiene una teoría de la acción adoptada que se basa en principios y preceptos, los que se ajustan a los antecedentes intelectuales y a nuestros compromisos. Sin embargo, hay diferencias frente a cuál se recurre en momentos de estrés, siendo inconsistentes en la forma de actuar.

Es importante gestionar el negocio y adaptarlo a las nuevas tendencias sean redes sociales o colaborativas, las cuales acompasen los cambios que ha sufrido el entorno y por ende el negocio, y que a la vez converja con los objetivos de la empresa; con ello se va a facilitar el trabajo promocionando herramientas comunes, similares a las que se utilizan a diario.

Por otro lado, se ve una mayor cercanía con el cliente por medio de los diferentes medios de comunicación; como también presenta una mayor coherencia dentro y fuera de la empresa. Siempre y cuando se tome en cuenta que la empresa ha de mostrar las mismas "facetas" en la organización, interrelacionada con la cultura de la corporación.

Los canales de comunicación interna deben ser útiles para que las organizaciones puedan evaluar la calidad de sus procesos internos, por dicha razón, los líderes y directores deberán utilizarla para poder identificar problemas operacionales y diagnosticar la situación, para poder encontrar soluciones y métodos de estudio de feedback; para entender cómo está fluyendo la información relevante, de acuerdo al desempeño y las necesidades de la organización. 
"Hay que considerar como potenciales usuarios de información a todos aquellos miembros de la organización en sus diversos escalones de responsabilidad, evitando restringir la información a los presidentes, directivos y altos ejecutivos de la organización". (Gómez-Aguilar, M.2007, op. cit p.13).

Generalmente, ocurren disfonías en el sentido que la mayoría de los miembros que trabajan dentro de la empresa quieren recibir más información de la que envían.

Ocurre también que cuanto más alto es el nivel hacia donde se envían los mensajes, existe menor probabilidad de seguimiento.

Si bien los canales internos son relevantes, ocurre que en muchas ocasiones las mejores fuentes de información, son aquellas que son más cercanas a los empleados y las que dan peores resultados son las más lejanas.

La información debe fluir en varios niveles:

- Estratégica/Directiva: en el sentido que los mandos medios y gerentes, tienen un rol fundamental en la comunicación interna, trabajando como emisores de objetivos y estrategias de negocios, como también de receptores.

- Operativa: los jefes utilizan la comunicación para poder interactuar con su equipo, brindar un feedback, recompensas, motivar a los mismos, fomentar el trabajo en equipo y el sentido del grupo, asicomo también la comunicación de visión, valores y objetivos.

- Funcional: en este sentido se ve a la comunicación transversal a los procesos de negocio, como un área posicionada en forma estratégica dentro de la empresa, la cual presenta gran visibilidad interna y apoya a las diferentes áreas internas. (DeciDes, 2013, recurso electrónico).

En ocasiones, ocurre que la alta dirección proporciona información que es menos oportuna y de menor calidad que otras fuentes clave de información, a pesar que los miembros prefieren tener más información de los canales formales que de la vía clandestina. Si bien, se encuentran satisfechos, les gusta recibir más comunicación frente a frente especialmente de la alta gerencia.

Los miembros se satisfacen con fluentes los cuales demuestran confianza; mientras que otros limitan la apertura y reconocimientos.

Cuanto mayor es la distancia entre las fuentes de información y los receptores se percibe menor información, y menos oportunidades para requerir más información, hay menos seguimiento, la calidad de la información es peor, 
se usa más la vía clandestina y son peores las relaciones interpersonales. (Kreps, 1995, pp. 234-240).

Para gestionar de forma eficiente la información, se debe:

- Conocer las necesidades internas de información, en cada uno de los sectores de la empresa, ciclos de servicios, procesos y procedimientos internos.

- Visualizar la fluidez de la información, precisión de la misma, interacción y el nivel de comunicación.

- Entrenar a los miembros de la empresa en el uso de la información y su mejor gestión, visualizarla como un recurso intangible de gran valor, transversal a los procesos organizacionales.

- Utilizarla con el afán de contribuir a los procesos internos, pudiendo modernizar o perfeccionar las actividades de la organización.

- Conocer las necesidades de información externa, y poder satisfacerla por medio de un proceso de ida y vuelta, que se pueda retroalimentar.

Como comunicadores internos se debe evitar que la buena comunicación bloquee el aprendizaje; por tal razón, se debe hacer que los colaboradores sean parte del proceso, ya que, se desea tener un control unilateral, maximizar ganancias y minimizar pérdidas, reprimir sentimientos negativos y ser tan racionales como fuese posible y, mediante ello, podemos trazar metas y evaluar su cumplimiento. Esto conlleva al anti-aprendizaje, ya que en muy raras ocasiones reflexionamos en lo que damos por sentado.

Por eso es relevante que el aprendizaje sea de doble circuito donde se puedan cuestionar nuestras acciones $y$, gracias a ello, mejorarlas.

Cuando se plantea un proyecto dentro de la organización y en este caso un proyecto de mejora en la comunicación organizacional, se debería fijar objetivos que cooperen al logro del plan, si no se tiene en claro lo que se desea realizar y qué metas alcanzar no será posible desarrollar un proyecto coherente.

No obstante, en ocasiones ocurre que no se definen correctamente estos objetivos porque no están claramente delimitados, siendo poco realistas y precisos.

El acrónimo SMART traducido a español se define como "inteligente", o sea que deben ser eficaces y estar correctamente formulados, para que nos allanen el camino.

El afán es poder definir objetivos que sean medibles, se puedan lograr, sean específicos y que se delimiten en un plazo de tiempo esperado y fijado. Se desea que el objetivo sea desafiante y 
difícil de aplicar pero se debe hacer hincapié en que este no debe ser idílico.

- S-Específico: En este sentido se pone énfasis en que sea lo más detallado y concreto posible, si bien, parece complejo el objetivo debe expresar de la mejor forma qué es lo que se desea hacer, de qué manera, cómo, quién lo hará y para qué, o sea que debe precisar de forma clara y específica el efecto que espera lograr.

- M - Mensurable: Debe ser medible, la meta se debe cuantificar por medio de indicadores; los criterios de medición deberán ser objetivos y factibles. Muchas veces es complejo llegar a una medición que acompase con el objetivo; sin embargo, es deseable aproximarse lo más posible al impacto causado por el mismo. A su vez es de suma relevancia contar con descriptores sobre qué intentamos lograr, quién lo va a realizar, qué cambio se espera, el tiempo estimado para lograrlo, o sea que contendrá todos aquellos elementos de cantidad, claridad y recursos que se van a utilizar para poder evaluarlos. Tenemos que saber cuándo cumplimos con el objetivo, medir significa seguimiento y control, lo que supone "trackear" el objetivo.

- A - Alcanzable: el objetivo debe tener un equilibrio entre lo que desea- mos hacer y los recursos con los que contamos, debe ser un reto para la empresa, ser ambicioso y desafiante, pero a la vez deberá contar con expectativas que sean realistas para que la empresa pueda cumplirlo, y así no generar desaliento. Por ello, es fundamental a la hora de plantearlos ir paso a paso, alinearlos con la estrategia y debe ser posible ajustarlos en caso que hayan cambios en el entorno.

R - Relevante: Debe ser importante y nosotros debemos querer lograrlo, siendo realistas y enfatizando en resultados, estando alineado a la estrategia organizacional; esto supone que debe ser factible con los recursos y limitaciones con los que cuenta la empresa. Puede ocurrir que un objetivo sea relevante, pero no sea posible costear la inversión en ese momento, por ello es menester tener en cuenta la situación de la empresa.

- T - A tiempo: Se deberá establecer una línea de tiempo, es ideal marcar un calendario con cada uno de ellos, definiendo el plazo estimado de consecución tomando en cuenta el futuro y el escenario deseado. Asimismo deberá contar con métricas que nos validen a conocer y obtener datos en el momento que sean de utilidad. 
El otro aspecto a destacar es que deben establecer prioridades, ser fáciles de memorizar para poder captar y motivar a los miembros de la organización, deben dar sustento a la asignación de recursos facilitando el proceso y evaluando resultados a priori y posteriori, disminuyendo así la incertidumbre de los resultados que se pretenden lograr en el futuro, pudiendo evaluar la eficacia de varios factores (sea por departamento, actividad, trabajador).

Por ejemplo, la empresa "XX" desea que más personas visiten su página Web

S: ¿Cuántas visitas más deseamos conseguir? ¿A quién nos enfocamos? ¿Con qué finalidad?

M: Al menos un $30 \%$ más de visitar, o sea pasar de 1.000 visitas mensuales a por lo menos 1.300.

A: Es ambicioso y realista a la vez, contamos con los recursos y personal para lograrlo. Podemos hacer promociones y publicidad, estamos a tiempo, tenemos stock para hacer frente.

R: La idea de potenciar las visitas es dar a conocer la nueva colección y que esto genere un impacto en las ventas de indumentarias para regalos de navidad.

T: Al 30 de noviembre de 2018.

\section{CONCLUSIONES}

Como menciona Robbins (2012, p. 20), "las organizaciones exitosas actuales deben impulsar la innovación y dominar el arte del cambio, o se volverán candidatas a extinción". Por ello, el beneficio primordial para la empresa será tener una atención diferencial, con el fin de generar valor agregado para poder resaltar frente a la competencia, y con ello, captar la atención de los clientes y lograr que los mismos elijan a la empresa en frente a su competencia.

\section{Motivación = Fuerza de la motivación * Valor de la meta + Expectativa}

Por medio de estos valores se podrá mejorar la motivación, ya que una o dos de las variables que componen la ecuación van a acrecentarse en impacto y valor.

La "Cohesión grupal" servirá para lograr mayor apoyo entre los compañeros hacia un mismo objetivo generando un funcionamiento positivo.

Asimismo, y siguiendo en línea a estos factores se debe tener en cuenta que los objetivos deberán estar delimita- 
dos de forma correcta y ser SMART; objetivos muy ambiciosos pueden acarrear desmotivación, mientras que objetivos muy básicos pueden conllevar a logro rápido y desilusión. 


\section{REFERENCIAS}

BARNETT, M. L., JERMIER, J. M., \& LAFFERTY, B. A. (2006). Corporate reputation: The definitional landscape. Corporate reputation review, 9(1), 26-38.

CARRIÓN, S. (2011). Curso de Practitioner en PNL: la nueva técnica para el cambio y el éxito. Ediciones Obelisco.

Cortina, A. (2005). Ética de la empresa, no sólo responsabilidad social. El país, 20.

COSTA, Joan (2009): Imagen corporativa en el Siglo XXI. 2da. edición, 4ta. reimpresión, La Crujía: Buenos Aires.

DECIDES (26 de abril de 2013). Colaboradores Alineados y Comprometidos, una estrategia empresarial. Recuperado de: https://decides. $\mathrm{cl} /$ web/colaboradores-alineados-y-comprometidos-una-estrategia-empresarial

DERES (s/f). Pacto Global. Recuperado de: https://deres.org.uy/pacto-global/

FIOL, C. M. (1991). Managing culture as a competitive resource: An identity-based view of sustainable competitive advantage. Journal of management, 17(1), 191-211.

FIOL, C. M., \& HUFF, A. S. (1992). Maps for managers: Where are we? Where do we go from here?. Journal of management studies, 29(3), 267285.

Giner, A. (2008). Las empresas transnacionales y los derechos humanos. Lan harremanak: Revista de relaciones laborales, (19), 67-88.

HUXLEY, Aldous (2009). Las puertas de la percepción. Cielo e infierno. Traducción de Miguel de Hernani. Barcelona: Editorial Edhasa.

Kotler P, de Madariaga, J, Miranda, Flores Zamora, M, Bowen, J, Makens, J. (2011). Marketing Turístico. España, 5ta edición: Pearson.

Kreps, G. (1995) La comunicación en las organizaciones, Estados Unidos: Addison - Wesley Iberoamericana O'CONNOR, J. y SEYMOUR, J. (2007). Introducción a la PNL. Ed.: Urano, Barcelona.

PACTO MUNDIAL. (29 de noviembre de 2017). Principio 1: Referente a Derechos Humanos. Red Española. Recuperado de: https://www. pactomundial.org/2017/11/principio-1/

Pérez, Y. \& Coutín, A. (2005). La gestión del conocimiento: un nuevo enfoque en la gestión empresarial. ACIMED, 13(6) Recuperado en 21 de septiembre de 2019, de http://scielo.sld.cu/scielo. 
php?script=sci_arttext\&pi$d=$ S1024-94352005000600004\&lng=es\&tlng=es.

Robbins, S y Judge, A (2013). Traducción por Pineda, S. Comportamiento organizacional. Edición Nro. 15. Pearson, México.

RODRIGUEZ, C., (2016). El liderazgo sustentable. Cómo liderar, sentir y cambiar el mundo. 1era edición. EDICON, Ciudad Autónoma de Buenos Aires: Argentina.
Volpentesta (2011) Gestión de la Responsabilidad Social Empresaria. "Visión, misión, estrategia y políticas de la empresa. 2da edición. Librería Editorial: Osmar D. Buyatti. Buenos Aires: Argentina.

Tangarife-Pedraza, M. A. (2008). De la responsabilidad de la empresa y los Derechos Humanos. International Law: Revista Colombiana de Derecho Internacional, (12), 145-181. 